\title{
High-level tolerance to triclosan may play a role in Pseudomonas aeruginosa antibiotic resistance in immunocompromised hosts: evidence from outbreak investigation
}

\author{
Silvia D'Arezzo ${ }^{1}$, Simone Lanini ${ }^{1,3^{*}}$, Vincenzo Puro', Giuseppe Ippolito ${ }^{1}$ and Paolo Visca ${ }^{2}$
}

\begin{abstract}
Background and methods: Pseudomonas aeruginosa is a major infectious threat to immunocompromised patients. We recently reported a fatal epidemic of multidrug-resistant $P$. aeruginosa in an onchoematology unit, linked to massive contamination of a triclosan-based disinfectant. The aim of this study is to evaluate the antimicrobial activity of triclosan and chlorhexidine digluconate against the epidemic strain of $P$. aeruginosa, to confirm the hypothesis that the soap dispenser acted as a continuous source of the infection during the outbreak, and to explore the potential role of triclosan in increasing the level of resistance to selected antibiotics. Susceptibility tests and time-kill assays for disinfectans were performed using two commercial formulations containing triclosan and chlorhexidine digluconate, respectively. Antibiotic susceptibility testing was performed by the broth microdilution method.

Findings: The $P$. aeruginosa epidemic strain exhibited an extremely high level of triclosan resistance (apparent MIC $=2,125 \mathrm{mg} / \mathrm{L}$ ), while it was markedly susceptible to chlorhexidine digluconate (apparent MIC $=12.5 \mathrm{mg} / \mathrm{L}$ ). Upon gradual adaptation to triclosan, the epidemic strain survived for a long period (> 120 h) in the presence of 3,400 $\mathrm{mg} / \mathrm{L}$ (equivalent to $1.6 \times \mathrm{MIC}$ ) of triclosan, concomitantly increasing the resistance to six antibiotics that are typical substrates of drug efflux pumps of the resistance nodulation division family. This effect was reversed by efflux pump inhibitors.

Conclusions: The epidemic P. aeruginosa strain was resistant to triclosan and its previous exposure to triclosan increases antibiotic resistance, likely through active efflux mechanisms. Since $P$. aeruginosa can become tolerant to elevated triclosan concentrations, the use of triclosan-based disinfectants should be avoided in those healthcare settings hosting patients at high risk for $P$. aeruginosa infection.
\end{abstract}

\section{Background}

The Gram-negative, rod shaped, aerobic bacterium Pseudomonas aeruginosa is a leading cause of severe infections in hospitalized subjects [1]. Colonization of the hospital environment, which may also involve antiseptic solutions, is a well-established mechanisms of $P$. aeruginosa persistence and spread in healthcare setting $[2,3]$.

\footnotetext{
* Correspondence: simone.lanini@inmi.it

'National Institute for Infectious Diseases "Lazzaro Spallanzani", I.R.C.C.S., Rome, Italy

Full list of author information is available at the end of the article
}

We recently described an outbreak of multidrug-resistant (MDR) P. aeruginosa infection which occurred in a oncohematology unit in Italy [3]. The outbreak involved 5 patients, 3 of whom died. Isolates from all 5 cases of infection showed an identical antibiotic resistance pattern (i.e. resistant to fluoroquinolones and aminoglycosides and susceptible to beta-lactams), all belonged to sequence type 175 , and showed identical pulsotype and RAPD type, forming a single epidemiological cluster. We were also able to trace back the source of infection to a common soap dispenser which was found to be heavily contaminated with the same $P$. aeruginosa isolates infecting the cases. This soap dispenser used to be
C Biomed Central

(C) 2012 D'Arezzo et al; licensee BioMed Central Ltd. This article is published under license to BioMed Central Ltd. This is an Open Access article distributed under the terms of the Creative Commons Attribution License (http://creativecommons.org/licenses/by/2.0), which permits unrestricted use, distribution, and reproduction in any medium, provided the original work is properly cited. 
manually refilled with commercial preparations of either $4 \%$ clorexidine digluconate or $0.5 \%$ triclosan in 3 months rotation; at the time infections set in, the dispenser contained the triclosan soap.

Triclosan is an antiseptics commonly used in hospitals because of the broad antimicrobial activity [4]. However, concern has been raised over triclosan efficacy since some bacterial species are endowed with intrinsic resistance to this compound [4]. P. aeruginosa is resistant to triclosan as the consequence of the expression of multiple mechanisms, including active efflux from the cells mediated by the resistance nodulation division (RND) family of efflux pumps [5], the presence of a triclosanresistant enoyl-acyl carrier protein reductase (FabV) [6], outer membrane impermeability, and the expression of a triclosan-specific pump (TriABC-OpmH) $[7,8]$. Some of these mechanisms are also associated with the development of resistance to some clinically significant antibiotics, raising the additional concern that, under certain circumstances, inappropriate use of triclosancontaining disinfectants may select for resistance against clinically useful drugs $[9,10]$. The aim of this study was to evaluate the activity of two commercial disinfectants containing triclosan and chlorhexidine digluconate against the epidemic $P$. aeruginosa strain, in order to confirm the hypothesis that the soap dispenser containing triclosan may have actually acted as a continuous source of the infection during the outbreak [3], and to explore the potential role played by triclosan in enhancing the level of resistance to selected antibiotics.

\section{Methods}

\section{Bacterial isolates}

Six different $P$. aeruginosa strains were isolated throughout the outbreak investigation, as previously reported [3]. P. aeruginosa strain L2 was isolated in the triclosancontaining soap dispenser and isolates L3 and L4 from water outlets in patients rooms (environmental isolates). P. aeruginosa isolates 10, 11 and 13 were isolated from patients' biological specimens (clinical isolates). Isolates L2, 10, 11 and 13 all belonged to sequence type 175 and were indistinguishable by pulsotyping, RAPD and MLST analysis, while isolates L3 and L4 showed a distinct type [3]. After primary isolation, isolates were sub-cultured for 4-6 times in Mueller-Hinton (MH, Oxoid, Milan, Italy) agar, and then stored frozen in $\mathrm{MH}$ broth.

\section{Disinfectants}

Two commercial disinfectants were used in this study, designated product $A$ and product $B$, whose composition (\% w/v in water) is: (A) 0.5\% 2,4,4'-trichloro-2'-hydroxydiphenyl-ether (triclosan), 10\% sodium lauryl-myristyl ether sulfate, $3 \%$ coconut glycerol, $2.5 \%$ coconut amido propyl betaine, and 1.5\% alcohol ethoxylate; $(B) 4 \%$ chlorhexidine gluconate, $15 \%$ polysorbate $20,5 \%$ coconut diethanolamide, $4 \%$ alkyl polyglucoside, $1 \%$ polyethylene glycol (PEG)-150, 1\% isopropyl alcohol, $0.1 \%$ essence, and $0.005 \%$ E 124 .

\section{Susceptibility tests for disinfectants}

Taking into account the very poor solubility of triclosan in aqueous solutions [11], the antimicrobial susceptibility tests and the time kill assay were performed using the commercial soap formulations. In the commercial product $A$, triclosan is well soluble (it is $5,000 \mathrm{mg} / \mathrm{L}$ ), and its addition to $\mathrm{MH}$ broth (that was adequately concentrated to have a final concentration of $1 \times \mathrm{MH}$ broth in all assays) did not result in any precipitation of the disinfectant.

$P$. aeruginosa was grown to the early stationary phase in $\mathrm{MH}$ broth. Bacterial cultures were diluted to 0.5 MacFarland turbidity in MH broth $\left(\sim 5 \times 10^{8} \mathrm{CFU} / \mathrm{ml}\right)$. This suspension was diluted 1:10 $\left(\sim 5 \times 10^{7} \mathrm{CF} / \mathrm{mL}\right)$ in $\mathrm{MH}$ broth, and $10 \mu \mathrm{l}$ were used as inoculum in 96-well microtiter plates (Costar, Cambridge, Massachusetts) containing $190 \mu \mathrm{l}$ of serial two-fold dilutions of the disinfectant soaps in $1 \times \mathrm{MH}$ broth. To test the highest concentration of triclosan $(4,250 \mathrm{mg} / \mathrm{L}$, which corresponds to the minimum soap dilution), $20 \mu \mathrm{l}$ of $10 \times$ concentrated MH broth were mixed with $170 \mu \mathrm{l}$ of undiluted triclosan soap (product A) containing $5,000 \mathrm{mg} / \mathrm{L}$ triclosan and $10 \mu \mathrm{l}$ of bacterial inoculum $\left(\sim 2.5 \times 10^{6}\right)$. All other dilutions were prepared by combining appropriate volumes of $10 \times$ concentrated $\mathrm{MH}$ broth, triclosan soap, sterile water, and the standard $10-\mu l$ bacterial inoculum.

To test the susceptibility of $P$. aeruginosa to chlorexidine digluconate (product $B$ ), standard two-fold dilutions of the disinfectant were generated in $1 \times \mathrm{MH}$ broth and inoculated with $\sim 2.5 \times 10^{6} \mathrm{CFU} / \mathrm{mL}$ in 96 -well microtiter plates (Costar).

Since the disinfectant soaps were commercial mixtures of triclosan or chlorhexidine with other ingredients which may or may not be inert, the observed minimal inhibitory concentrations (MICs) were called "apparent MICs" (a-MICs). The a-MIC was expressed as the minimal concentration of the disinfectant in the commercial formulation causing no growth after 48 -h incubation at $37^{\circ} \mathrm{C}[12,13]$, with chlorhexidine $>50 \mathrm{mg} / \mathrm{L}$ [14] and triclosan $>128 \mathrm{mg} / \mathrm{L}$ [13] as resistance breakpoints. Each experiment was performed in triplicate.

\section{Determination of the bactericidal activity for disinfectants} $P$. aeruginosa L2 suspensions $\left(\sim 5 \times 10^{7} \mathrm{CFU} / \mathrm{mL}\right)$ were made in $\mathrm{MH}$ broth supplemented with either product $A$ (triclosan range: $1,062-4,250 \mathrm{mg} / \mathrm{L}$ ) or product $B$ (chlorhexidine digluconate range: $12.5-1,250 \mathrm{mg} / \mathrm{L}$ ), and incubated at $37^{\circ} \mathrm{C}$. The two products were diluted 
in $\mathrm{MH}$ broth, prepared at the appropriate concentration in order to compensate for the addition of an appropriate volume of disinfectant and a constant bacterial inoculum. At defined intervals, aliquots were neutralized by rapid serial dilution in $\mathrm{MH}$ broth supplemented with $0.5 \%$ lecithin and $4 \%$ polysorbate 20 [15], plated on $\mathrm{MH}$ agar plates, and examined after 24$\mathrm{h}$ incubation at $37^{\circ} \mathrm{C}$ for determination of viable counts.

\section{Adaptation to high triclosan concentration}

The frozen stock of $P$. aeruginosa L2 was readapted to triclosan by passages in gradually increasing triclosan concentrations. Inocula of $\sim 5 \times 10^{5} \mathrm{CFU} / \mathrm{mL}$ were serially cultured in stepwise increasing triclosan concentrations in $\mathrm{MH}$ broth at $37^{\circ} \mathrm{C}$, starting with $1 / 2$ the MIC and doubling the concentration at each passage until evidence of full growth.

\section{Antimicrobial susceptibility testing}

The susceptibility of triclosan-adapted and unadapted $P$. aeruginosa to a panel of antibiotics which are typically exported by RND efflux pumps [16], namely tetracycline, ciprofloxacin, amikacin, levofloxacin, carbenicillin and chloramphenicol (all from Sigma-Aldrich) was determined by the broth microdilution method according to the CLSI guidelines [12] either with or without the RND efflux pump inhibitor phenyl-arginine- $\beta$ naphthylamide $(\mathrm{PA} \beta \mathrm{N})$ or the protonophore carbonyl cyanide m-chlorophenylhydrazone (CCCP) (SigmaAldrich) at the predetermined concentrations of $50 \mu \mathrm{M}$ and $100 \mu \mathrm{M}$, respectively. Each experiment was performed in triplicate.

\section{Results}

Susceptibility to triclosan and chlorhexidine digluconate Elevated triclosan resistance $(\mathrm{a}-\mathrm{MIC}=2,125 \mathrm{mg} / \mathrm{L}$ for isolates $10,11,13$ and $\mathrm{L} 2$; a-MIC $=4,250 \mathrm{mg} / \mathrm{L}$ for isolates L3 and L4) was observed for both clinical and environmental $P$. aeruginosa isolates tested after primary isolation and serial passages in $\mathrm{MH}$ broth. Conversely, all isolates were markedly susceptible to chlorhexidine digluconate $(\mathrm{a}-\mathrm{MIC}=12.5 \mathrm{mg} / \mathrm{L})$.

Determination of the bactericidal activity for disinfectants Time-kill kinetics of the two disinfectants were determined for the P. aeruginosa L2 soap isolate, regarded to as the prototypic epidemic strain.

Chlorhexidine digluconate at $\geq 125 \mathrm{mg} / \mathrm{L}$ exerted a rapid bactericidal effect $\left(>3 \log _{10}\right.$ reduction of viable counts) within 5 min of contact with $P$. aeruginosa L2 cells. This concentration is far below the actual concentration, $40,000 \mathrm{mg} / \mathrm{L}$, provided by the manufacturer in the commercial product B (Figure 1, upper panel).
The triclosan-containing product $A$ was much less effective in killing P. aeruginosa L2 cells (Figure 1, lower panel). Only the highest triclosan concentration achievable in $\mathrm{MH}$ broth $(4,250 \mathrm{mg} / \mathrm{L}$, equivalent to $2 \times$ MIC) exerted strong bactericidal activity within $3 \mathrm{~h}$ of contact with $P$. aeruginosa cells $\left(>6 \log _{10}\right.$ reduction of $\mathrm{CFU} / \mathrm{ml})$. When exposed to triclosan concentrations of 1,6 $\times$ MIC $(3,400 \mathrm{mg} / \mathrm{L})$, L2 cells were killed during the initial $6 \mathrm{~h}\left(>3 \log _{10}\right.$ reduction of $\left.\mathrm{CFU} / \mathrm{ml}\right)$, but the residual population could then survive for a long period $(>120 \mathrm{~h})$ in the presence of this concentration of the disinfectant. At triclosan concentration of 2,125 and $1,062 \mathrm{mg} / \mathrm{L}$ (equivalent to $1 \times$ and $0,5 \times \mathrm{MIC}$, respectively) $\sim 1 \log _{10}$ reduction of cell viability was observed relative to the inoculum size at $24 \mathrm{~h}$, but afterwards growth was restored and the bacterial population reached the same density as the control in triclosan-free medium after $120 \mathrm{~h}$.

\section{Adaptation to triclosan results in increased levels of antibiotic resistance}

In $P$. aeruginosa, triclosan resistance is mediated at least by 4 efflux pumps, belonging to the RND family (MexAB-OprM, MexCD-OprJ, MexEF-OprN, and MexXY-OprM), which can also drive active extrusion of various antibiotics $[5,10]$. Therefore, we tested the susceptibility to six antibiotics (tetracycline, ciprofloxacin, amikacin, levofloxacin carbenicillin and chloramphenicol) typically exported via RND pumps in unadapted and triclosan-adapted $P$. aeruginosa L2 cells (Table 1 ). We observed that the efflux pump inhibitors $P A \beta N$ or $\mathrm{CCCP}$, at concentrations which do not affect bacterial growth, decreased the MIC of tetracycline, ciprofloxacin, amikacin, levofloxacin, carbenicillin and chloramphenicol by a factor 2-4 in triclosan-unadapted $P$. aeruginosa cells. Remarkably, adaptation of $P$. aeruginosa L2 to sub-lethal concentrations of triclosan (up to $3,400 \mathrm{mg} / \mathrm{L}$, see Methods and Table 1) resulted in a 2-fold increase of MICs for all six antibiotics. Efflux pump inhibitors (PA $\beta N$ or CCCP) restored antibiotic susceptibility of the triclosan-adapted isolate to the same or even higher level than the unadapted isolate (2-4 fold reduction of MICs, Table 1), suggesting that active antibiotic efflux was enhanced in P. aeruginosa cells following triclosan exposure.

\section{Discussion}

A $P$. aeruginosa strain, which eventually caused a fatal epidemic cluster, was able to contaminate and likely replicate within a triclosan-soap dispenser. Triclosan is an anionic, lipophilic compound that is very poorly soluble in water [11]. However, previous researchers have reported that various solubilizers, such as the surfactant sodium lauryl sulphate, increase the solubility of 

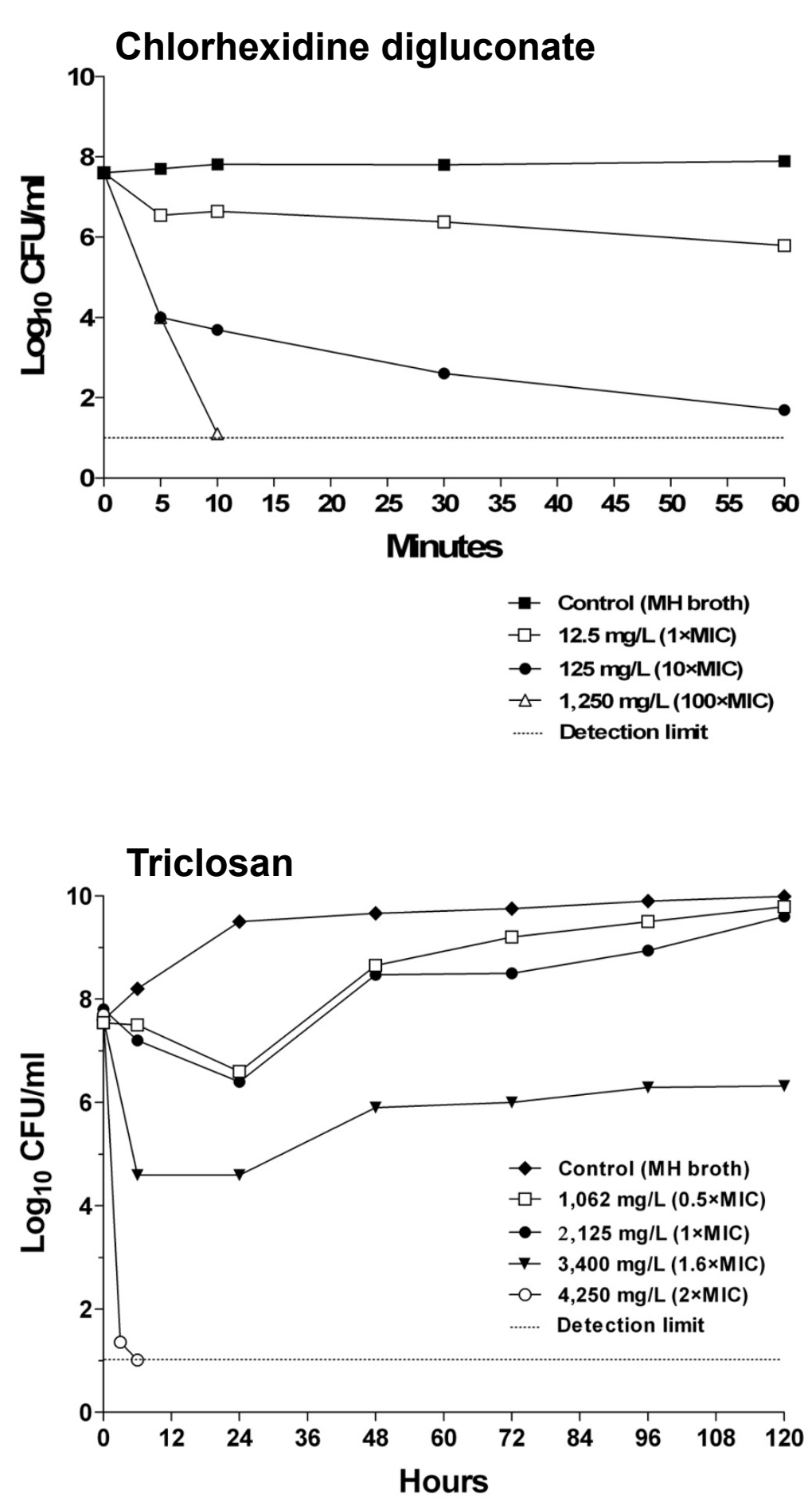

Figure 1 Time-kill kinetics of $P$. aeruginosa L2 in MH broth supplemented with chlorhexidine digluconate (product $B$, upper panel) or triclosan (product $A$, lower panel) at given concentrations.

triclosan from 80- to 6,000-fold. Micellar solubilization and the formation of either salts or complexes are postulated as possible mechanisms for the increase in the solubility of triclosan [11]. In this study, we decided to perform the antimicrobial susceptibility tests and the time kill assays using the commercial soap product $A$, in which triclosan $(5,000 \mathrm{mg} / \mathrm{L})$ is kept in solution by $10 \%$ sodium lauryl-myristyl ether sulfate. Assuming that this 
Table 1 Antibiotic susceptibility in unadapted and triclosan-adapted P.aeruginosa

\begin{tabular}{|c|c|c|c|c|c|c|}
\hline \multirow[t]{2}{*}{ P. aeruginosa L2 } & \multicolumn{6}{|c|}{ Antibiotic MIC (mg/L) ${ }^{b}$} \\
\hline & TET & CIP & AMK & LVX & CAR & $\mathrm{CHL}$ \\
\hline Unadapted & 32 & 16 & 4 & 8 & 128 & 128 \\
\hline Unadapted plus PABN (50 $\mu \mathrm{M})$ & 16 & 4 & 2 & 2 & 64 & 64 \\
\hline Unadapted plus CCCP $(100 \mu \mathrm{M})$ & 16 & 8 & 2 & 4 & 64 & 64 \\
\hline Adapted ${ }^{a}$ to $3,400 \mathrm{mg} / \mathrm{L}$ triclosan & 64 & 32 & 8 & 16 & 256 & 256 \\
\hline Adapted ${ }^{a}$ to $3,400 \mathrm{mg} / \mathrm{L}$ triclosan plus PABN $(50 \mu \mathrm{M})$ & 32 & 8 & 4 & 4 & 64 & 64 \\
\hline 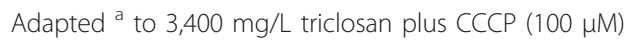 & 32 & 16 & 4 & 8 & 64 & 64 \\
\hline
\end{tabular}

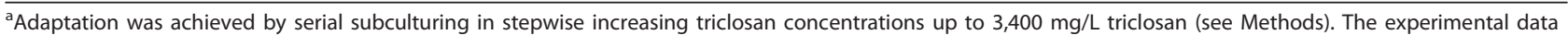
were confirmed by three independent replicates

${ }^{\mathrm{b}}$ Breakpoint criteria were as follows. TET: susceptible, $\leq 4 \mathrm{mg} / \mathrm{L}$; intermediate, $8 \mathrm{mg} / \mathrm{L}$; resistant, $\geq 16 \mathrm{mg} / \mathrm{L} ;$ CIP: susceptible, $\leq 1 \mathrm{mg} / \mathrm{L}$; intermediate, $2 \mathrm{mg} / \mathrm{L}$; resistant, $\geq 4 \mathrm{mg} / \mathrm{L} ;$ AMK: susceptible, $\leq 16 \mathrm{mg} / \mathrm{L}$; resistant, $\geq 32 \mathrm{mg} / \mathrm{L}$; LVX: susceptible, $\leq 2 \mathrm{mg} / \mathrm{L}$; intermediate, $4 \mathrm{mg} / \mathrm{L} ;$ resistant, $\geq 8 \mathrm{mg} / \mathrm{L} ;$ CAR: susceptible, $\leq$ $128 \mathrm{mg} / \mathrm{L}$; intermediate, $256 \mathrm{mg} / \mathrm{L}$; resistant, $\geq 512 \mathrm{mg} / \mathrm{L} ; \mathrm{CHL}$ : susceptible, $\leq 8 \mathrm{mg} / \mathrm{L}$; intermediate, $16 \mathrm{mg} / \mathrm{L} ;$ resistant, $\geq 32 \mathrm{mg} / \mathrm{L}$ [12] MIC minimal inhibitory concentration; $P A \beta N$ RND pump inhibitor phenyl-arginine- $\beta$-naphthylamide; $C C C P$ protonophore carbonyl cyanide $\mathrm{m}$ chlorophenylhydrazone; TET tetracycline; CIP ciprofloxacin; AMK amikacin; LVX levofloxacin; CAR carbenicillin; CHL chloramphenicol

surfactant, or any other additive of product $A$, may have increased the effectiveness of triclosan in killing $P$. aeruginosa, this would paradoxically imply that the actual $P$. aeruginosa resistance to triclosan alone would be higher than that observed in our experimental system.

Determination of the bactericidal activity for triclosan confirmed that the epidemic strain could have contaminated the soap dispenser most probably because of its remarkable tolerance to triclosan. In fact this epidemic strain was originally isolated at relatively high concentration $\left(5 \times 10^{4} \mathrm{CFU} / \mathrm{mL}\right)$ from the dispenser containing the triclosan-based commercial soap (product $A$ ) for healthcare workers' hand washing [3]. These findings provide additional biological evidence to confirm our previous hypothesis, based on molecular identity between patients' and soap P. aeruginosa isolates [3], that the contaminated soap dispenser may have acted as the primary source of infection. We can also suppose that the activity of triclosan in the commercial formulation was reduced by partial inactivation of triclosan by micellar entrapping on inclusion which may have reduced the amount of free triclosan available in solution.

Conversely, the chlorhexidine-based disinfectant, which was employed in rotation of 3 months with triclosan in the oncohematology unit [3], had strong and fast bactericidal effect at concentrations far below those recommended by the manufacturer for practical use.

Another interesting result was that the level of resistance to some antibiotics, namely tetracycline, fluoroquinolones, aminoglycosides, carbenicillin and chloramphenicol, was increased by previous adaptation of $P$. aeruginosa to triclosan. As reported in other studies $[10,16]$, triclosan may induce the expression of multidrug efflux systems belonging to RND family in $P$. aeruginosa. These systems are relatively nonspecific in substrate recognition and enables bacteria to pump out numerous chemically unrelated substances, such as antibiotics and biocides. Here, we report that the pattern of increasing resistance to antibiotics was likely to be driven by activation of efflux pumps belonging to the RND family; in fact, the RND efflux pump inhibitor $P A \beta N$ and the protonophore CCCP restored the baseline antibiotic susceptibility in the triclosan-adapted strain. Thus, while the link between triclosan usage and development of clinically significant clinical resistance to antibiotics remains controversial $[9,10]$, here we provide evidence that previous exposure to triclosan elevates the level of antibiotic resistance. Although the differences in MICs obtained for the different antibiotics are small (2fold), they provide a clear evidence of the overall trend towards increasing antibiotic resistance associated with adaptation to tricosan. Increased resistance was also observed for those antibiotics to which $P$. aeruginosa L2 cells showed elevated baseline resistance. As noted for carbenicillin, adaptation to triclosan converted the susceptible phenotype ( $\mathrm{MIC} \leq 128 \mathrm{mg} / \mathrm{L})$ into intermediate resistant $(\mathrm{MIC}=256 \mathrm{mg} / \mathrm{L})$.

To the best of our knowledge, this is the first time that such a high tolerance to triclosan is documented in a clinical $P$. aeruginosa isolate, since previous studies on both uncharacterized and type (PAO1) $P$. aeruginosa strains reported actual MICs of $\sim 1,000 \mathrm{mg} / \mathrm{L}$ [17].

Some issues limit the generalizability our findings. Firstly, our data come from a single epidemic event, and therefore they may not apply in the same way to seemingly similar episodes in other institutions or to general clinical practices as biological features of the outbreak strain (e.g. virulence, infectivity, and/or baseline resistance patterns) and/or local situation (e.g. infection control policies, mode of transmission, and/or risk factors) may be unique. Secondly, we could not quantify the actual triclosan concentration in the triclosan-based commercial soap formulation - as it was in the soap dispenser at the time of the outbreak - since the oncohematology unit was closed due to legal issue, 
and the only soap sample obtained was insufficient for chemical analysis. Therefore we cannot rule out that accidental dilution (below the commercial concentration) of triclosan soap due to refilling procedure might have played a preeminent role in facilitating the growth of $P$. aeruginosa in the dispenser and the eventual spreading of the infection. Finally, we only performed phenotypic assays, and we cannot provide a genetic basis for the remarkably high resistance to triclosan of the $P$. aeruginosa outbreak strain. Intrinsic outer membrane impermeability, resistance of the FabV enoyl-acyl carrier protein reductase, expression of an additional triclosan-specific pump (TriABC-OpmH) or other unknown mechanisms could have acted synergistically with efflux in determining the unprecedented triclosan resistance observed our isolates.

Despite these limitations, this study provide biological evidence to confirm that an unprecedented environmental source, which was identified as a triclosan soap dispenser, actually acted as a continuous source of infection during the outbreak. In addition, we provide hints that exposure to sub-lethal concentration of triclosan may contribute to antibiotic resistance in P. aeruginosa clinical isolates.

\section{Conclusions}

Since certain strains of $P$. aeruginosa can grow in the presence of triclosan concentrations similar to those present in commercial soap formulations, triclosanbased disinfectants can incidentally be contaminated and act as a continuous source of $P$. aeruginosa in healthcare settings. The concentration of triclosan-based disinfectants meant to be used in those facilities admitting patients at high risk for $P$. aeruginosa infection should be reassessed.

\section{Funding}

This work was supported by a grant from Ministero della Salute- Ricerca Corrente 2008.

\begin{abstract}
Abbreviations
P. aeruginosa: Pseudomonas aeruginosa; RND: resistance nodulation and cell division; w/v: weight/volume (as concentration measure); CFU: colony forming unit; MIC: minimal inhibitory concentration; PA $\beta N$ : pump inhibitor phenyl-arginine- $\beta$-naphthylamide; CCCP: pump inhibitor protonophore carbonyl cyanide m-chlorophenylhydrazone; $\mathrm{MH}$ : Mueller-Hinton; $\mu \mathrm{M}$ : micro molar; mg: milligrams; $\mathrm{mL}$ : millilitre; ${ }^{\circ} \mathrm{C}$ : Celsius degree; L: litre.
\end{abstract}

\section{Author details}

'National Institute for Infectious Diseases "Lazzaro Spallanzani", I.R.C.C.S., Rome, Italy. 'Department of Biology, University "Roma Tre", Rome, Italy. ${ }^{3}$ Dipartimento di Epidemiologia e Ricerca pre-clinica, Istituto Nazionale per le Malattie Infettive, Via Portuense 292, 00149 Roma, Italia.

\section{Authors' contributions}

SDA carried out the phenotypic assays and drafted the manuscript. SL and VP participated in the design of the study and obtained samples during outbreak investigations. VP and Gl conceived of the study, and participated in its design and coordination and helped to draft the manuscript. All authors read and approved the final manuscript.

\section{Competing interests}

The authors declare that they have no competing interests.

Received: 19 August 2011 Accepted: 19 January 2012

Published: 19 January 2012

\section{References}

1. Kunz AN, Brook I: Emerging resistant Gram-negative aerobic bacilli in hospital-acquired infections. Chemotherapy 2010, 56:492-500.

2. Engelhart S, Krizek L, Glasmacher A, Fischnaller E, Marklein G, Exner M: Pseudomonas aeruginos outbreak in a haematology-oncology unit associated with contaminated surface cleaning equipment. J Hosp Infect 2002, 52:93-98.

3. Lanini S, D'Arezzo S, Puro V, Martini L, Imperi F, Piselli P, Montanaro M, Paoletti S, Visca P, Ippolito G: Molecular epidemiology of a hospital outbreak driven by a contaminated disinfectant-soap dispenser. PLOS One 2011, 6:e17064.

4. McDonnell G, Russell AD: Antiseptics and disinfectants: activity, action, and resistance. Clin Microbiol Rev 1999, 12:147-179.

5. Poole K: Multidrug efflux pumps and antimicrobial resistance in Pseudomonas aeruginos and related organisms. J Mol Microbiol Biotechnol 2001, 3:255-264.

6. Zhu L, Lin J, Ma J, Cronan JE, Wang H: Triclosan resistance of Pseudomonas aeruginos PAO1 is due to FabV, a triclosan-resistant enoylacyl carrier protein reductase. Antimicrob Agents Chemother 2010, 54:689-698.

7. Mima T, Joshi S, Gomez-Escalada M, Schweizer HP: Identification and characterization of TriABC-OpmH, a triclosan efflux pump of Pseudomonas aeruginos requiring two membrane fusion proteins. $J$ Bacteriol 2007, 189:7600-7609.

8. Schweizer HP: Triclosan: a widely used biocide and its link to antibiotics. FEMS Microbiol Lett 2001, 202:1-7.

9. Walsh SE, Maillard JY, Russell AD, Catrenich CE, Charbonneau DL, Bartolo RG: Development of bacterial resistance to several biocides and effects on antibiotic susceptibility. J Hosp Infect 2003, 55:98-107.

10. Chuanchuen R, Beinlich K, Hoang TT, Becher A, Karkhoff-Schweizer RR, Schweizer HP: Cross-resistance between triclosan and antibiotics in Pseudomonas aeruginos is mediated by multidrug efflux pumps: exposure of a susceptible mutant strain to triclosan selects $n f x$ mutants overexpressing MexCD-OprJ. Antimicrob Agents Chemother 2001, 45:428-432.

11. Grove C, Liebenberg W, du Preez JL, Yang W, de Villiers MM: Improving the aqueous solubility of triclosan by solubilization, complexation, and in situ salt formation. J Cosmet Sci 2003, 54:537-550.

12. Clinical and Laboratory Standard Institute: Performance standards for antimicrobial susceptibility testing, twenty-first informational supplemen Wayne: CLSI document M 100-S21; 2011.

13. Orsi GB, Tomao P, Visca P: In vitro activity of commercially manufactured disinfectants against Pseudomonas aeruginos. Eur J Epidemiol 1995, 11:453-457.

14. Nakahara $\mathrm{H}$, Kozukue $\mathrm{H}$ : Isolation of chlorhexidine-resistant Pseudomonas aeruginos from clinical lesions. J Clin Microbiol 1982, 15:166-168.

15. Kampf $G$, Shaffer M, Hunte $C$ : Insufficient neutralization in testing a chlorhexidine-containing ethanol-based hand rub can result in a false positive efficacy assessment. BMC Infect Dis 2005, 5:48.

16. Chuanchuen R, Karkhoff-Schweizer RR, Schweizer HP: High-level triclosan resistance in Pseudomonas aeruginos is solely a result of efflux. Am J Infect Control 2003, 31:124-127.

17. Bhargava HN, Leonard PA: Triclosan: applications and safety. Am J Infect Control 1996, 24:209-218.

doi:10.1186/1756-0500-5-43

Cite this article as: D'Arezzo et al:: High-level tolerance to triclosan may play a role in Pseudomonas aeruginosa antibiotic resistance in immunocompromised hosts: evidence from outbreak investigation. BMC Research Notes 2012 5:43. 